\title{
Las TIC como herramientas cognitivas para la investigación
}

\author{
Oscar Yecid Aparicio Gómez ${ }^{1}$ \\ Olga Lucía Ostos Ortiz ${ }^{2}$
}

Recibido: 20-02-2018

Aceptado: 23-04-2018

El uso de las TIC como herramienta cognitiva aplicada a la investigación requiere un estudio de las mejores alternativas para que los estudiantes puedan disponer de estas amplificadoras de la mente, que estimulen el pensamiento crítico y puedan construir el conocimiento a través del aprendizaje colaborativo. Gros (2013) afirma que la tecnología tiene sentido para mejorar el aprendizaje siempre y cuando se parta de una perspectiva constructivista a través de experiencias basadas en la interacción social, la participación activa y los entornos complejos.

Las TIC solo adquieren significado cuando se ordenan a metas que no son ellas en sí mismas, tales como el desarrollo integral de las personas mediante el uso de la tecnología en actividades como la investigación (Viejo, Cabezas, \& Martínez, 2013). Por tanto, los usos más eficaces de las TIC en las aulas son para acceder a la información y la interpretación, organización y representación del conocimiento personal (Jonassen, 2000). En ese sentido Coll, Mauri y Onrubia (2009) afirman que las TIC se emplean de manera específica para apoyar o amplificar las habilidades de conocimiento que se utilizan en procesos complejos de resolución de problemas. Y este es nuestro caso a la hora de usar las TIC como herramienta cognitiva para la investigación.

Las TIC digitales, ofrecen una serie de herramientas para la investigación que se caracterizan por la utilización simultánea y combinada de diversos lenguajes y formatos pudiendo mezclar además el sonido, imágenes estáticas y en movimiento, diversos tipos de lenguaje, sistemas de símbolos y de diversa representación Coll (2013). Por su parte, las

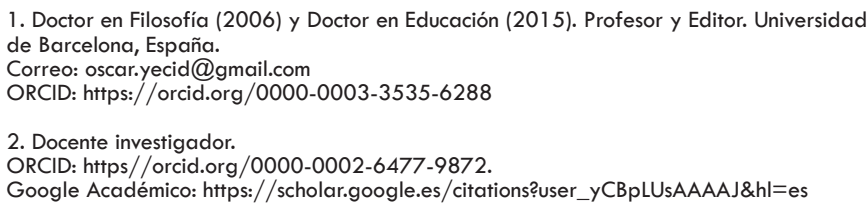


tecnologías móviles e inalámbricas permiten pensar en un modelo de aprendizaje en el que el aprendiz aprenda lo que tiene interés por aprender en diferentes momentos y contextos de actividad y pueda hacerlo cambiando con rapidez y facilidad de un contexto a otro (Loou y otros, 2010). Las características de las TIC digitales presentadas como aplicaciones de dispositivos móviles optimizan los resultados cognitivos de los estudiantes y promueven diversas iniciativas de investigación y profundización en los más diversos contextos (Pérez, 2013).

Se trata de considerar las posibilidades que ofrecen las TIC (Coll, Mauri y Onrubia, 2008b) para acceder y presentar, casos y problemas complejos; incluir materiales multimedia e hipermedia en los casos o problemas y en la presentación de los contenidos de apoyo a su estudio y abordaje; profundizar en la representación inicial del caso o problema y progresar en el planteamiento del mismo; comunicarse y colaborar en procesos de solución de problemas y de construcción del conocimiento.

Las TIC pueden considerarse como herramientas que permiten trascender las limitaciones cognitivas del procesamiento humano para ponerse así al servicio de los procesos investigativos en los diversos medios sociales (Azevedo, 2014). Las TIC, en el caso de las instituciones educativas, pueden ponerse a disposición de la comunidad educativa para motivar la innovación, compartir y estimular la carga cognitiva del escolar, procesar y clasificar la información recogida, así como para reinterpretar y resignificar los datos conseguidos mediante prácticas investigativas, y permitir la generación de hipótesis en el contexto del método científico, entre otras muchas funciones operativas (Ferreyra, 2014).

\section{La Plataforma Moodle como herramienta cognitiva}

El uso del aula virtual es una forma motivacional de incluir los objetos de estudio, las fases del trabajo y apoyo para el desarrollo de las tareas con el acompañamiento de los profesores (Aparicio, 2018). Este tipo de Plataforma ayuda en el desarrollo curricular para actualizar el lenguaje al que habitualmente se usa en la Sociedad del Conocimiento y a incluir talleres o elementos adicionales que aporte a una nueva visión de la investigación desde el ámbito escolar (de Almeida, Santos \& Porto 2016). 
Las diversas herramientas de las que se sirve la tecnología, facilitan el diseño de las unidades didácticas y modalidades de intervención sobre las actividades y tareas de los estudiantes (Konieczny, 2015). El enfoque colaborativo que bien se puede aprovechar tanto para estudiantes como para profesores, puede generar la construcción del conocimiento en espacios colaborativos. Las Plataformas proporcionan amplias fuentes de información a través de diversos objetos virtuales y foros de debate. (de la Calle et al., 2014) así, la comunidad científica puede beneficiarse cada vez más del uso de la tecnología para la promoción e implementación de acciones investigativas en ámbitos académicos.

En nuestro caso específico de estudio nos referiremos a la Plataforma Moodle $e^{3}$ como una Plataforma de gestión del aprendizaje diseñada como un sistema que permite la integración de aplicaciones externas e información dentro de una única Plataforma para crear ambientes virtuales de aprendizaje personalizado. Moodle se proporciona como un programa de código abierto gratuito, para usar, modificar y distribuir libremente bajo los términos de la Licencia GPL (General Public License). Moodle tiene certificación de compatibilidad con el estándar Learning Tool Interoperability (LTI) para la integración de aplicaciones para aprendizaje, y cumple con la especificación SCORM (Sharable Content Object Reference Model) para que puedan funcionar otros contenidos creados para la web.

En la página de Moodle ${ }^{4}$, los desarrolladores reconocen que el desenvolvimiento de esta Plataforma se ha llevado a cabo bajo la guía de la pedagogía de constructivismo social y el construccionismo ${ }^{5}$ (Aparicio, 2018). A través de la Plataforma Moodle se pretende que los aprendices construyan activamente nuevos conocimientos mientras interactúan con su entorno. (Tahull, 2016) todas las experiencias se contrastan con otras anteriores para formar nuevo conocimiento, privilegiando la interpretación sobre la transmisión de la información. Moodle promueve la construcción del conocimiento para que pueda socializarse, creando colaborativamente conocimientos con significados compartidos; así, las actividades y textos producidos dentro del grupo también ayudarán a definir la participación de cada persona en el equipo de trabajo.

Las herramientas proporcionadas por Moodle están centradas en el proceso educativo del estudiante, y en la construcción activa de sus

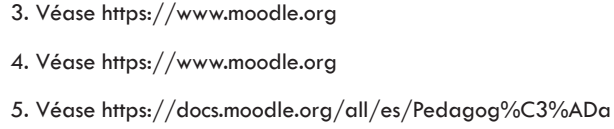


propios conocimientos mientras interactúa con sus pares o profesores. Moodle ofrece a esta comunidad educativa la creación colaborativa de "artefactos" compartidos con significados compartidos.

A través de las Plataformas Moodle puede reconocerse que la fuente de conocimiento fluye entre los miembros de la comunidad educativa de estudiante a profesor y viceversa, así como entre los mismos estudiantes, conectando así a todos según las necesidades de aprendizaje de cada uno o del grupo.

En el sitio web https://moodle.org se destacan cinco referentes disponibles en la Plataforma cuando se trata del constructivismo social, entendido como:

- Todos somos tanto profesores como estudiantes potenciales; en un entorno verdaderamente colaborativo somos las dos cosas.

- Aprendemos particularmente bien creando o expresando algo para que otros lo vean.

- Aprendemos mucho simplemente observando la actividad de nuestros pares.

- Entendiendo el contexto de otros podemos enseñar de un modo mejor.

- Un entorno de aprendizaje necesita ser flexible y adaptable para poder responder con rapidez a las necesidades de los participantes en su interior.

Aunque la Plataforma Moodle puede ser una herramienta con potencial cognitivo, ella dispone de otras herramientas específicas que enriquecen el proceso de aprendizaje, y que en el contexto de este trabajo de tesis doctoral hemos considerado su disposición estructural para usarlo en un contexto investigativo a nivel escolar como herramienta de recolección, análisis, interpretación de datos, así como para la publicación y socialización de los resultados obtenidos, en un contexto donde los estudiantes y quienes les acompañan puedan disfrutarlo al máximo. 
Las actividades de la Plataforma Moodle cubren diferentes funciones del proceso educativo. En cuanto al aprendizaje, se pueden identificar actividades que se orientan hacia la construcción de conocimiento a través de diversas modalidades: personal, en grupo o colaborativa. Igualmente, la comunicación de procesos de conocimiento o su evaluación encuentra en las actividades de la plataforma una manera que facilita la relación entre la comunidad educativa a nivel síncrono o asíncrono.

\section{Referencias bibliográficas}

Aparicio, O.Y. (2018). Las TIC como herramientas cognitivas. Revista Interamericana de Investigación, Educación y Pedagogía, RIIEP, 11(1). https://orcid.org/0000-0003-35356288

Azevedo, S. R. J. (2014). La composición histórica de la educación religiosa como componente curricular. Revista Interamericana de Investigación, Educación y Pedagogía, RIIEP, 7(1). DOI: https://doi.org/10.15332/s1657-107X.2014.0001.03

Coll, C., Mauri, T. y Onrubia, J. (2008b). Los entornos virtuales de aprendizaje basados en el análisis de casos y la resolución de problemas. En Coll, C. y Monereo, C. (Eds.). Psicología de la educación virtual. Aprender y enseñar con las Tecnologías de la Información y la Comunicación (pp. 213 - 232), Madrid: Morata.

de Almeida, R. R., Santos, M. F., \& Porto, J. C. (2016). Lectura de textos ficcionales y el enfoque escolar de literatura: contribuciones para una Pedagogía de la Elección. Revista Interamericana de Educación, Pedagogía y Estudios Culturales, 9(1), 35-51. DOI: https://doi.org/10.22490/25391887.1925

de la Calle, C. V., Malaver, M. O., Gallego, J. D. M., Rodríguez, M., Flórez, J. C., Henao, C. E. \& Saldaña, R. (2014). Aportes de los doctorados de educación en ciencia, tecnología y sociedad, desde la sistematización de sus investigaciones doctorales científicas y formativas, 2000-2010. Revista Interamericana de Investigación, Educación y Pedagogía, RIIEP, 7(1). DOI: https://doi.org/10.15332/s1657-107X.2014.0001.04

Ferreyra, H. A. (2014). Mesas Socioeducativas para la Inclusión y la Igualdad. Un programa "De todos con todos". Una experiencia en construcción. Revista Interamericana de Investigación, Educación y Pedagogía, RIIEP, 7(2). DOI: https://doi. org/10.15332/s1657-107X.2014.0002.01

Gros, B. (2013). La educación más allá de la escuela: del espacio-lugar al espacio- nodo. En Rodríguez Illera, J. L. (Comp.) (2013). Aprendizaje y educación en la sociedad digital. Barcelona: Universitat de Barcelona. DOI: 10.1344/106.000002060 
Jonassen, D. H. (2000). Computers as Mindtools for Schools: Engaging Critical Thinking. New Jersey: Prentice - Hall.

Konieczny, P. (2015). Lorenzo García Aretio: bases, mediaciones y futuro de la educación a distancia en la sociedad digital. Revista Interamericana de Investigación, Educación y Pedagogía, RIIEP, 8(1). DOI: https://doi.org/10.15332/s1657107X.2015.0001.08

Pérez, T. H. P. (2013). Aproximaciones al estado de la cuestión de la investigación en educación y derechos humanos. Revista Interamericana de Investigación, Educación y Pedagogía, RIIEP, 6(1). DOI: https://doi.org/10.15332/s1657-107X.2013.0001.05

Tahull, J. (2016). Modernidad, educación y género. El proyecto inacabado. Revista Interamericana de Investigación, Educación y Pedagogía, RIIEP, 9(2), 159-178. DOI: https://doi.org/10.22490/25391887.1947

Viejo, C. M., Cabezas, I. L., \& Martínez, M. D. J. I. (2013). Las redes de académicas en la docencia universitaria. Revista Interamericana de Investigación, Educación y Pedagogía, RIIEP, 6(2). DOI: https://doi.org/10.15332/s1657-107X.2013.0002.03 